\title{
投喂频率对长鯺篮子鱼幼鱼生长性能、 肌肉粗营养及血清生化指标的影响
}

\author{
方可菲 ${ }^{1,2}$ ，刘鉴毅 ${ }^{1,2}$ ，王 妤 ${ }^{2}$, 冯广朋 ${ }^{1,2}$ ， \\ 邹 雄 ${ }^{2}$, 郝长杰 ${ }^{1,2}$, 余炎方 ${ }^{1,2}$, 孙雪娜 ${ }^{1,2}$
}

(1. 上海海洋大学, 水产科学国家级实验教学示范中心, 上海 201306;

2. 中国水产科学研究院东海水产研究所, 长江口渔业生态重点实验室, 上海 200090)

\begin{abstract}
摘 要: 为探究不同投喂频率对长魦篮子鱼 (Siganus canaliculatus) 幼鱼生长及生理的影响, 分别设置 4 个投 喂频率 $\left(1\right.$ 次 $\cdot d^{-1} 、 2$ 次 $\cdot d^{-1} 、 3$ 次 $\cdot d^{-1} 、 4$ 次 $\left.\cdot d^{-1}\right)$, 依次标记为 $F 1 、 F 2 、 F 3 、 F 4$ 组, 每组设置 3 个平行, 在微 流水养殖桶 (有效容积 $125 \mathrm{~L}$ ) 中养殖 16 周。结果显示: 1) 生长指标: 各组长鳀篮子鱼幼鱼体质量及体长随养 殖时间增加均呈线性上升趋势, F3 与 F4 组生长趋势相近; 饵料系数和摄食率 $\mathrm{F} 3$ 组均为最高, 且显著高于 F1 组 $(P<0.05) ; F 4$ 组肝体比显著高于 $F 1$ 组 $(P<0.05), F 1$ 组肠体比与肠长比显著高于 $\mathrm{F} 4$ 组 $(P<0.05), F 3$ 、 $\mathrm{F} 4$ 组肥满度显著高于 F1、F2 组 $(P<0.05)$ 。2) 肌肉粗营养: $\mathrm{F} 1$ 组肌肉中水分含量显著高于其他 3 组 $(P<$ $0.05)$, 而肌肉粗蛋白含量显著低于其余 3 组 $(P<0.05) ; \mathrm{F} 2 、 \mathrm{~F} 3$ 组肌肉粗灰分含量显著高于 $\mathrm{F} 1 、 \mathrm{~F} 4$ 组 $(P<$ $0.05)$ 。3 $)$ 血清生化指标: F2、F3 组血清总蛋白含量显著高于 F1、F4 组 $(P<0.05)$; 而 F1、F4 组血清白蛋白含 量显著高于 $\mathrm{F} 2 、 \mathrm{~F} 3$ 组 $(P<0.05)$; 甘油三酯的含量随投喂频率增加显著增加 $(P<0.05)$; F4 组高密度脂蛋白 胆固醇含量最高, 显著高于 $\mathrm{F} 1 、 \mathrm{~F} 2$ 组 $(P<0.05)$, 且 $\mathrm{F} 4$ 组低密度脂蛋白胆固醇含量显著低于其他 3 组 $(P<$ $0.05) ; \mathrm{F} 3$ 组谷草转氨酶活性及谷丙转氨酶活性显著低于其他 3 组 $(P<0.05)$ 。结果表明,投喂频率在 3 次 $\mathrm{d}^{-1}$ 时长鳍篮子鱼幼鱼的生长效益最佳。
\end{abstract}

关键词: 长鯺篮子鱼; 投喂频率; 生长; 肌肉粗成分; 血清生化指标 中图分类号: S 968.1

文献标志码: A

长鯺篮子鱼 (Siganus canaliculatus) 隶属于鲈 形目 (Perciformes), 篮子鱼科 (Siganidae), 篮子鱼 属, 属暖水性近海鱼类,在我国主要分布于东海、 南海和台湾海域 ${ }^{[1]}$ 。长鯺篮子鱼以藻类为食, 在 养殖过程中可起到清洁网箱网衣的作用,对养殖 环境有正向的生态价值,因此成为我国重要的海 洋经济鱼类之一。近年来, 海南、广东、福建沿海 的长鯺篮子鱼网箱养殖逐渐增多并趋于规模 化 $^{[2]}$ 。实际养殖生产中, 常通过以低频率投喂高 营养密度日粮或以高频率投喂低营养密度日粮 的投喂模式来提高经济养殖鱼类的生长效益 ${ }^{[3]}$,
但较低的投喂频率可能会导致养殖动物饥饿、相 互攻击和自相残杀 ${ }^{[4]}$, 而过高的投喂频率则会在 一定程度上影响鱼体的健康 ${ }^{[5]}$ 。

鱼类生长通常会受覀料的类型与规格、投喂 频率、投喂率及鱼吸收养分能力等因素影响 ${ }^{[6]}$ 。 近年来研究发现,投喂频率是影响鱼类生长性能 的关键因素之一 ${ }^{[7]}$ 。了解投喂频率对鱼类生长 的影响,有助于在养殖实践中提高鱼类的生长性 能; 通过研究鱼类各项生理指标受投喂频率的影 响情况, 还可评估鱼体的健康状况, 为防治各种 鱼类疾病提供参考依据 ${ }^{[8]}$ 。因此, 设置科学的投

收稿日期: $2020-02-14$

基金项目：国家重点研发计划(2019YFD0900405)；上海市科技兴农种业项目 [沪农科种字(2016)第 2-3 号]; 上海 市青年科技英才扬帆计划(17YF1425700)

作者简介：方可菲(1994一), 女, 硕士研究生, 主要从事水产养殖研究。E-mail :caven_fang@ 163. com

通信作者：刘鉴毅,研究员。E-mail:liujy@ ecsf. ac.cn 
喂频率对于鱼类养殖生产至关重要 ${ }^{[9]}$ 。

本研究以长鯺篮子鱼幼鱼为研究对象, 对不 同投喂频率下其生长指标、肌肉粗营养成分及血. 清生化指标等进行测定, 对各投喂频率下鱼的生 长效益进行评估, 旨在为长鯺篮子鱼及同属其他 鱼类的生产管理提供相关参考数据和资料。

\section{1 材料与方法}

\section{1 实验用鱼及饲料}

实验用鱼为福建省东山县近海捕获的野生 样本, 运至中国水产科学研究院东海水产研究所 福建福鼎基地, 在直径 $6 \mathrm{~m}$ 、深 $2 \mathrm{~m}$ (有效容积 $1000 \mathrm{~L}$ ) 的圆形暂养池中暂养 4 周, 使其适应实 验环境。暂养期间每天定时定点投喂 2 次 (7:00 和 17:00) 实验饲料。

实验所用饲料购自福建海大饲料有限公司 (饲料名称:篮子鱼膨化配合饲料), 暂养期间饲 料规格为 1 号。饲料粗成分: 粗蛋白 $40.5 \%$ 、粗 脂肪 $5.2 \%$ 、粗纤维 $3.39 \%$ 、粗灰分 $15.1 \%$ 、赖氨 酸 $2.21 \%$ 、水分 $10.25 \%$ 、总磷 $1.53 \%$ 。

\section{2 实验设计及饲养管理}

选择 1200 尾大小一致、鱼体外部无明显损 伤、初始平均体质量为 $(4.14 \pm 0.74) \mathrm{g}$ 的长鯺篮 子鱼幼鱼, 随机分养于 12 个养殖桶中 (桶直径 $1.5 \mathrm{~m}$ 、高 $0.7 \mathrm{~m}$, 有效容积 $125 \mathrm{~L}$ )。实验期间, 采 用表观饱食投喂, 设置 4 个投喂频率分别为 1 次 $\cdot d^{-1}$ (F1 组)、2 次 $\cdot d^{-1}$ (F2 组)、3 次 $\cdot d^{-1}$ (F3 组) 及 4 次 $\cdot d^{-1}$ ( F4 组), 每组设置 3 个平 行。养殖周期为 2019 年 7-11 月共 16 周。各组 投喂频率和投喂时间见表 1 。

实验过程中每次投喂至饱食状态, 投喂结束 $30 \mathrm{~min}$ 后收集每缸的残饵, 置于烘箱 $65{ }^{\circ} \mathrm{C}$ 烘干 并称重。实验期间记录每次投喂饵料质量及残 饵质量。养殖用水为经过沉淀砂滤的新鲜海水。 实验期间养殖水体各项水质指标为: 盐度 19
21 , 水温 $25 \sim 30{ }^{\circ} \mathrm{C}$, 溶解氧含量 $>5 \mathrm{mg} \cdot \mathrm{L}^{-1}$ 、氨 氮浓度 $<0.5 \mathrm{mg} \cdot \mathrm{L}^{-1}, \mathrm{pH}$ 值 $6.8 \sim 7.2$ 。整个养 殖过程采用微流水并 $24 \mathrm{~h}$ 连续充气, 日换水 1 次, 换水率 $100 \%$ 。

\section{3 生长数据测量方法及计算}

每隔 4 周对每组实验用鱼进行体质量与体 长的测量。每组随机选取 30 尾鱼, 禁食 $24 \mathrm{~h}$, 用 麻醉剂 ( MS-222,100 mg - $\mathrm{L}^{-1}$ ) 麻醉后进行测量。 增重率、体质量变异系数及体长变异系数根据每 次测量实验用鱼的体质量与体长计算可得。特 定生长率、饵料系数、摄食率、成活率在实验结束 时进行测量计算。

生长指标的计算公式如下:

特定生长率 $(\%)=\left(\ln W_{t}-\ln W_{0}\right) / t \times$ $100 \%$;

饵料系数 $=I /\left(W_{t}-W_{0}\right)$;

摄食率 $(\%)=I /\left[t \times\left(W_{0}+W_{t}\right) / 2\right] \times$ $100 \%$;

成活率 $(\%)=N_{t} / N_{0} \times 100 \%$;

增重率 $(\%)=\left(W_{t}-W_{0}\right) / W_{0} \times 100 \%$;

体质量变异系数 $(\%)=\mathrm{SD} / W \times 100 \%$;

体长变异系数 $(\%)=\mathrm{SD} / L \times 100 \%$; 式中: $W_{0} 、 W_{t}$ 分别为实验开始和结束时长鯺篮子 鱼幼鱼的体质量 $(\mathrm{g}) ; t$ 为实验天数 $(\mathrm{d}) ; I$ 为实验 期间总摄食量 $(\mathrm{g}) ; N_{t} 、 N_{0}$ 分别为实验结束时鱼的 尾数和实验开始时鱼的尾数; SD 为标准差; $W 、 L$ 为鱼的体质量 $(\mathrm{g})$ 、体长 $(\mathrm{cm})$ 。

\section{4 形态指标测量方法}

每组随机取 10 尾实验用鱼称量体质量及内 脏质量, 再分别称取肝胰脏、脾脏及肠的质量 (精 确到 $0.0001 \mathrm{~g}$ ), 用于计算脏体比、肝体比、脾体 比和肠体比; 并测量肠及鱼体长度, 计算出肠长 比。

形态指标计算公式如下:

脏体比 $(\%)=W_{\mathrm{V}} / W_{\mathrm{B}} \times 100 \%$;

表 1 长鯺篮子鱼幼鱼投喂时间和投喂频率

Tab. 1 Feeding time and frequency of juvenile $S$. canaliculatus

\begin{tabular}{|c|c|c|c|c|c|}
\hline \multirow{2}{*}{$\begin{array}{l}\text { 组别 } \\
\text { Group }\end{array}$} & \multicolumn{5}{|c|}{ 投喂时间和频率 Feeding time and frequency } \\
\hline & $7: 00$ & $10: 20$ & 12:00 & $13: 40$ & 17:00 \\
\hline F1 & 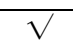 & & & & \\
\hline $\mathrm{F} 2$ & V & & & & V \\
\hline F3 & V & & V & & V \\
\hline F4 & V & V & & $V$ & $V$ \\
\hline
\end{tabular}


肝体比 $(\%)=W_{\mathrm{H}} / W_{\mathrm{B}} \times 100 \%$;

脾体比 $(\%)=W_{\mathrm{S}} / W_{\mathrm{B}} \times 100 \%$;

肠体比 $(\%)=W_{\mathrm{I}} / W_{\mathrm{B}} \times 100 \%$;

肠长比 $(\%)=L_{\mathrm{I}} / L \times 100 \%$;

肥满度 $\left(\mathrm{g} \cdot \mathrm{cm}^{-3}\right)=W_{\mathrm{B}} / L^{3} \times 100 \%$;

式中: $W_{\mathrm{V}} 、 W_{\mathrm{H}} 、 W_{\mathrm{S}} 、 W_{\mathrm{I}}$ 分别为内脏质量、肝胰脏质 量、脾脏质量及肠道质量 $(\mathrm{g}), W_{\mathrm{B}}$ 为鱼体质量 $(\mathrm{g}) ; L$ 为鱼体长 $(\mathrm{cm}), L_{\mathrm{I}}$ 为鱼肠道长 $(\mathrm{cm})$ 。

\section{5 体成分指标测量方法}

内脏数据测定后, 在鱼背鯺基部取适量去皮 肌肉,保存在 $-80{ }^{\circ} \mathrm{C}$ 冰箱用于鱼体粗营养成分指 标的测定。采用直接干燥法测定水分含量 $(\mathrm{GB} / \mathrm{T}$ 5009.3-2003); 凯氏定氮法测定粗蛋白质含量 (GB/T 5009. 5-2003) ; 索氏抽提法测定粗脂肪含 量 ( GB/T 5009. 6-2003); 灼烧称重法测定灰分含 量( GB/T 5009.4-2003)。

\section{6 血清生化指标测定方法}

每组随机取实验用鱼 15 尾, 分成 3 个平行, 用 $1 \mathrm{~mL}$ 注射器采集鱼尾静脉血, 将每个平行 5 尾鱼尾静脉血混合后在 $4{ }^{\circ} \mathrm{C}$ 冰箱内静置 $2 \mathrm{~h}$, 再 以 $3000 \mathrm{r} \cdot \mathrm{min}^{-1}$ 冷冻离心 $15 \mathrm{~min}$, 取出上层血 清, 保存于干冰中用于后续血清生化指标的测 定。血清生化指标: 总蛋白 (TP)、球蛋白 (ALB)、 血糖 (GLU)、高密度脂蛋白 (HDL)、低密度脂蛋 白 (LDL)、甘油三酯 ( TGK) 、总胆固醇 ( TCHO )、 碱性磷酸酶 (ALP)、谷草转氨酶 (AST) 和谷丙转 氨酶( ALT) 均采用南京建成试剂盒进行测定。

\section{7 统计分析方法}

所有实验数据采用平均值 \pm 标准差 $($ mean \pm
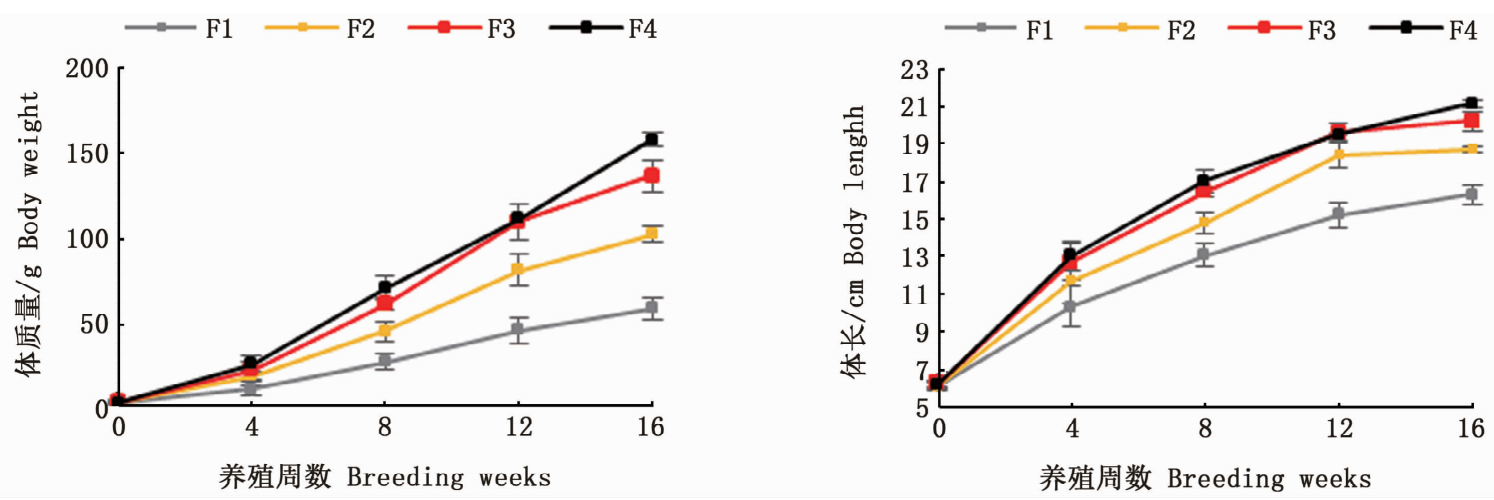

图 1 不同投喂频率下长鯺篮子鱼幼鱼体质量、体长变化情况

Fig. 1 Body weight and length dynamics of juvenile $S$. canaliculatus under different feeding frequencies 

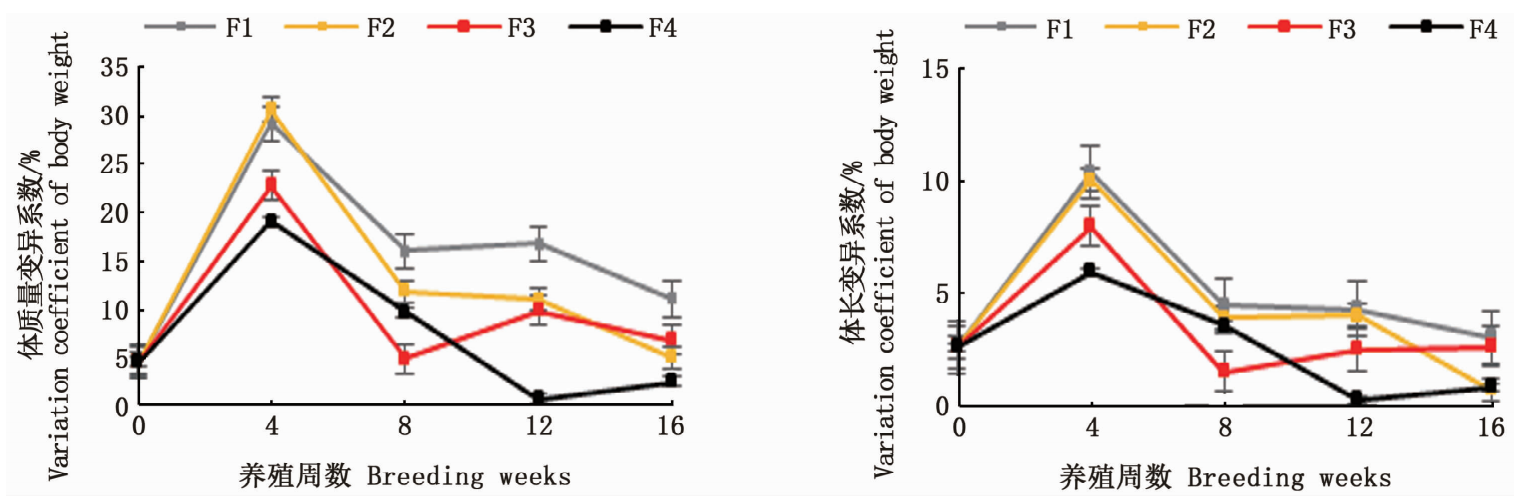

图 2 不同投喂频率下长鯺篮子鱼幼鱼的体质量及体长变异系数

Fig. 2 Variation coefficient of body weight and body length

of juvenile $S$. canaliculatus under different feeding frequencies

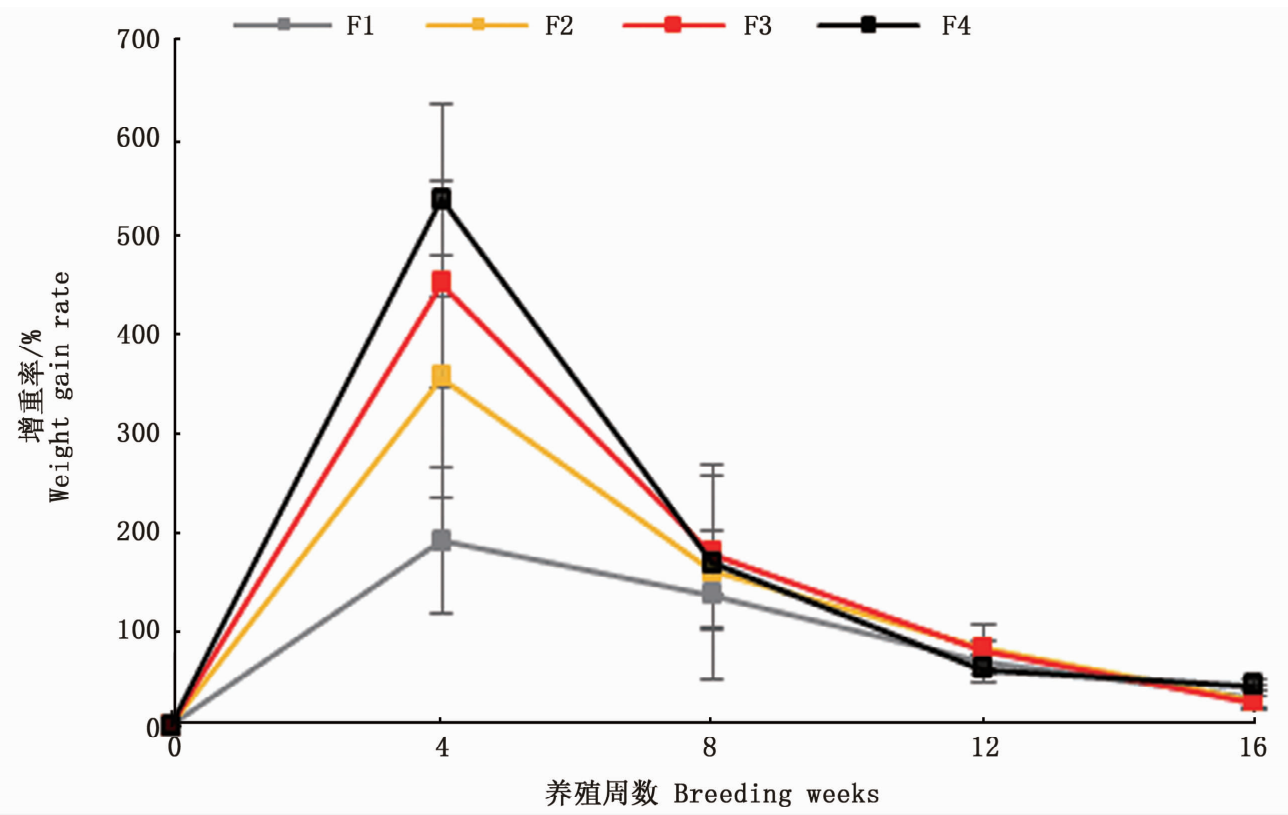

图 3 不同投喂频率下长鯺篮子鱼幼鱼的增重率变化

Fig. 3 Weight gain rate dynamics of juvenile $S$. canaliculatus under different feeding frequencies

表 2 投喂频率对长鯺篮子鱼幼鱼生长指标的影响

Tab. 2 Effect of feeding frequency on growth indices of juvenile $S$. canaliculatus

\begin{tabular}{ccccc}
\hline \multirow{2}{*}{ 指标 Indices } & \multicolumn{4}{c}{ 组别 Treatment } \\
\cline { 2 - 5 } & \multicolumn{1}{c}{$\mathrm{F} 1$} & $\mathrm{~F} 2$ & $\mathrm{~F} 3$ & $\mathrm{~F} 4$ \\
\hline 特定生长率 $\left./ \% \cdot \mathrm{d}^{-1}\right)$ & $2.10 \pm 0.06^{\mathrm{d}}$ & $2.54 \pm 0.08^{\mathrm{c}}$ & $2.77 \pm 0.05^{\mathrm{b}}$ & $2.88 \pm 0.05^{\mathrm{a}}$ \\
Specific growth rate & $1.58 \pm 0.18^{\mathrm{b}}$ & $2.17 \pm 0.09^{\mathrm{ab}}$ & $2.31 \pm 0.30^{\mathrm{a}}$ & $2.03 \pm 0.32^{\mathrm{ab}}$ \\
摄食率 $\%$ Feeding rate & $1.15 \pm 0.14^{\mathrm{b}}$ & $1.48 \pm 0.07^{\mathrm{a}}$ & $1.55 \pm 0.20^{\mathrm{a}}$ & $1.35 \pm 0.21^{\mathrm{ab}}$ \\
饵料系数 Feed conversion ratio & $94.50 \pm 2.50^{\mathrm{a}}$ & $95.00 \pm 4.97^{\mathrm{a}}$ & $95.33 \pm 2.49^{\mathrm{a}}$ & $92.67 \pm 6.18^{\mathrm{a}}$ \\
存活率 $/ \%$ Survival rate &
\end{tabular}

注: 同行数据上标字母不同表示不同投喂频率组之间差异显著 $(P<0.05)$, 字母相同表示差异不显著 $(P>0.05)$

Note: Data in the same row with different superscript letters mean significant differences $(P<0.05)$, the same letter means that the difference is not significant $(P>0.05)$ 


\section{3 投喂频率对长鯺篮子鱼幼鱼肌肉基本成分 的影响}

由表 4 可知, F1 组的肌肉水分含量显著高于 其他 3 组 $(P<0.05)$, 但 $\mathrm{F} 1$ 组肌肉粗蛋白含量显 著低于其余 3 组 $(P<0.05)$; F2、F3 组肌肉粗灰 分含量显著高于 $\mathrm{F} 1 、 \mathrm{~F} 4$ 组 $(P<0.05)$ 。

\section{4 投喂频率对长鯺篮子鱼幼鱼血清生化指标 的影响}

由表 5 可知, F2、F3 组血清总蛋白含量显著 高于 $\mathrm{F} 1 、 \mathrm{~F} 4$ 组 $(P<0.05)$; 而 $\mathrm{F} 1 、 \mathrm{~F} 4$ 组血清白蛋 白含量显著高于 $\mathrm{F} 2 、 \mathrm{~F} 3$ 组 $(P<0.05)$; 甘油三酯 的含量随投喂频率增加显著增加 $(P<0.05) ; \mathrm{F} 4$ 组高密度脂蛋白胆固醇含量最高, 显著高于 $\mathrm{F} 1$ 、 $\mathrm{F} 2$ 组 $(P<0.05)$, 而 F4 组低密度脂蛋白胆固醇 含量则显著低于其他 3 组 $(P<0.05)$; F3 组谷草 转氨酶活性及谷丙转氨酶活性显著低于其他 3 组 $(P<0.05)$ 。

\section{3 讨论}

\section{1 投喂频率对长鯺篮子鱼幼鱼生长的影响}

投喂频率是影响鱼体生长的重要因素。有 研究表明,适当增加投喂频率可显著提高鱼类的 特定生长率, 但而后继续增加投喂频率, 鱼类的 特定生长率却趋于稳定 ${ }^{[10]}$ 。本研究中, 各组鱼特
定生长率受投喂频率影响显著, 表现为随着投喂 频率的增加而显著上升, 这与对许氏平鲉

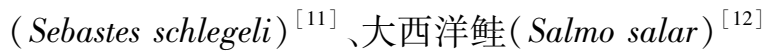
及瓦氏黄颡鱼 (Pelteobagrus vachelli $)^{[13]}$ 等的研究 结果一致。同时, 本研究中, $1 \sim 3$ 次 $\cdot \mathrm{d}^{-1}$ 投喂 频率组长鯺篮子鱼幼鱼的体质量、体长变化受投 喂频率影响显著, 但当投喂频率从 3 次 $\cdot \mathrm{d}^{-1}$ 增 加到 4 次 $\cdot \mathrm{d}^{-1}$ 时, 鱼体质量与体长不再显著变 化, 这与刘康和何金㻇 ${ }^{[14]}$ 对长吻鯔 (Leiocassis longirostris) 的研究结果相似; 且由增重率的变化 趋势可知, 前 4 周长鯺篮子鱼幼鱼受投喂频率影 响最为显著。

鱼类生长离散程度通常受养殖密度及摄食 量的影响;管敏等 ${ }^{[15]}$ 研究发现, 饱食投喂频率会 显著影响中华鲟 (Acipenser sinensis) 的生长离散现 象。本研究中, 前 4 周时, $2 \sim 4$ 次 $\cdot \mathrm{d}^{-1}$ 投喂频 率组间长鳍篮子鱼的体质量、体长变异系数有显 著差异,而 1 次 $\cdot \mathrm{d}^{-1}$ 与 2 次 $\cdot \mathrm{d}^{-1}$ 组间无显著差 异, 表明在 $0 \sim 4$ 周鱼体处于快速生长状态时, 饲 料投喂量的限制导致鱼群内部大个体鱼生长优 势更为明显,而增加饱食投喂频率可使不同大小 个体鱼均能摄人充足能量, 减少了鱼体生长离散 现象的发生。

表 3 投喂频率对长鯺篮子鱼幼鱼形态指标的影响

Tab. 3 Effect of feeding frequency on morphological indices of juvenile $S$. canaliculatus

\begin{tabular}{crrrr}
\hline \multirow{2}{*}{ 指标 Indices } & \multicolumn{4}{c}{ 组别 Treatment } \\
\cline { 2 - 5 } & \multicolumn{1}{c}{$\mathrm{F} 1$} & \multicolumn{1}{c}{$\mathrm{F} 3$} & $\mathrm{~F} 4$ \\
\hline 脏体比 $/ \%$ Viseraosomatic index & $11.24 \pm 0.51^{\mathrm{a}}$ & $12.11 \pm 0.78^{\mathrm{a}}$ & $12.34 \pm 1.38^{\mathrm{a}}$ & $12.44 \pm 1.26^{\mathrm{a}}$ \\
肝体比 $/ \%$ Hepatosmatic index & $1.75 \pm 0.35^{\mathrm{b}}$ & $2.09 \pm 0.34^{\mathrm{ab}}$ & $2.03 \pm 0.72^{\mathrm{ab}}$ & $2.84 \pm 0.61^{\mathrm{a}}$ \\
脾体比 $/ \%$ Ratio of spleen weight to body weight & $0.11 \pm 0.08^{\mathrm{a}}$ & $0.15 \pm 0.04^{\mathrm{a}}$ & $0.15 \pm 0.04^{\mathrm{a}}$ & $0.14 \pm 0.10^{\mathrm{a}}$ \\
肠体比 $/ \%$ Ratio of intestine weight to body weight & $2.69 \pm 0.43^{\mathrm{a}}$ & $2.21 \pm 0.41^{\mathrm{ab}}$ & $2.22 \pm 0.70^{\mathrm{ab}}$ & $1.69 \pm 0.25^{\mathrm{b}}$ \\
肠长比 $/ \%$ Ratio of intestine length to body length & $171.00 \pm 3.75^{\mathrm{a}}$ & $151.34 \pm 3.49^{\mathrm{ab}}$ & $156.83 \pm 7.66^{\mathrm{ab}}$ & $147.13 \pm 5.97^{\mathrm{b}}$ \\
肥满度 $/\left(\mathrm{g} \cdot \mathrm{cm}^{-3}\right)$ Condition factor & $1.35 \pm 0.03^{\mathrm{c}}$ & $1.55 \pm 0.05^{\mathrm{b}}$ & $1.65 \pm 0.05^{\mathrm{a}}$ & $1.66 \pm 0.03^{\mathrm{a}}$ \\
\hline
\end{tabular}

注: 同行数据上标字母不同表示不同投喂频率组之间差异显著 $(P<0.05)$, 字母相同表示差异不显著 $(P>0.05)$

Note: Data in the same row with different superscript letters mean significant differences $(P<0.05)$, the same letter means that the difference is not significant $(P>0.05)$

表 4 投喂频率对长鯺篮子鱼幼鱼肌肉基本成分的影响 (\%,湿重)

Tab. 4 Effect of feeding frequency on muscle composition of juvenile $S$. canaliculatus ( \%, wet weight)

\begin{tabular}{|c|c|c|c|c|}
\hline \multirow{2}{*}{ 指标 Indices } & \multicolumn{4}{|c|}{ 组别 Treatment } \\
\hline & $\mathrm{F} 1$ & $\mathrm{~F} 2$ & F3 & F4 \\
\hline 水分 Moisture & $76.27 \pm 0.10^{\mathrm{a}}$ & $71.61 \pm 1.63^{b}$ & $70.88 \pm 0.99^{b}$ & $71.78 \pm 1.85^{b}$ \\
\hline 粗蛋白 Crude protein & $17.76 \pm 0.42^{b}$ & $21.41 \pm 1.44^{\mathrm{a}}$ & $22.15 \pm 1.03^{\mathrm{a}}$ & $22.74 \pm 2.13^{\mathrm{a}}$ \\
\hline 粗脂肪 Crude lipid & $0.81 \pm 0.02^{\mathrm{a}}$ & $0.93 \pm 0.16^{\mathrm{a}}$ & $0.98 \pm 0.17^{\mathrm{a}}$ & $1.03 \pm 0.04^{\mathrm{a}}$ \\
\hline 粗灰分 Crude ash & $5.17 \pm 0.03^{b}$ & $6.07 \pm 0.04^{\mathrm{a}}$ & $6.00 \pm 0.21^{\mathrm{a}}$ & $3.46 \pm 0.23^{c}$ \\
\hline
\end{tabular}

注: 同行数据上标字母不同表示不同投喂频率组之间差异显著 $(P<0.05)$,字母相同表示差异不显著 $(P>0.05)$

Note: Data in the same row with different superscript letters mean significant differences $(P<0.05)$, the same letter means that the difference is not significant $(P>0.05)$ 
表 5 投喂频率对长鯺篮子鱼幼鱼血清生化指标的影响

Tab. 5 Effect of feeding frequency on serum biochemical indices of juvenile $S$. canaliculatus

\begin{tabular}{crrrr}
\hline \multirow{2}{*}{ 指标 Indices } & \multicolumn{4}{c}{ 组别 Treatment } \\
\cline { 2 - 5 } & \multicolumn{1}{c}{$\mathrm{F} 1$} & \multicolumn{1}{c}{$\mathrm{F} 2$} & $\mathrm{~F} 3$ \\
\hline 总蛋白 $/\left(\mathrm{g} \cdot \mathrm{L}^{-1}\right) \mathrm{TP}$ & $13.62 \pm 1.86^{\mathrm{b}}$ & $21.21 \pm 2.50^{\mathrm{a}}$ & $20.46 \pm 1.34^{\mathrm{a}}$ & $15.30 \pm 1.81^{\mathrm{b}}$ \\
白蛋白 $/\left(\mathrm{g} \cdot \mathrm{L}^{-1}\right) \mathrm{ALB}$ & $7.97 \pm 1.13^{\mathrm{c}}$ & $11.97 \pm 0.65^{\mathrm{a}}$ & $9.94 \pm 0.55^{\mathrm{b}}$ & $7.29 \pm 0.94^{\mathrm{c}}$ \\
葡萄糖 $/\left(\mathrm{mmol} \cdot \mathrm{L}^{-1}\right) \mathrm{GLU}$ & $9.83 \pm 2.37^{\mathrm{a}}$ & $10.35 \pm 1.74^{\mathrm{a}}$ & $10.13 \pm 2.03^{\mathrm{a}}$ & $9.29 \pm 0.22^{\mathrm{a}}$ \\
总胆固醇 $/\left(\mathrm{mmol} \cdot \mathrm{L}^{-1}\right) \mathrm{TCHO}$ & $22.11 \pm 0.13^{\mathrm{a}}$ & $21.50 \pm 2.23^{\mathrm{a}}$ & $23.51 \pm 2.10^{\mathrm{a}}$ & $23.75 \pm 1.24^{\mathrm{a}}$ \\
甘油三酯 $/\left(\mathrm{mmol} \cdot \mathrm{L}^{-1}\right) \mathrm{TG}$ & $7.33 \pm 0.10^{\mathrm{d}}$ & $8.64 \pm 0.32^{\mathrm{c}}$ & $9.63 \pm 0.47^{\mathrm{b}}$ & $11.28 \pm 0.70^{\mathrm{a}}$ \\
高密度脂蛋白胆固醇 $/\left(\mathrm{mmol} \cdot \mathrm{L}^{-1}\right) \mathrm{HDL}$ & $0.86 \pm 0.10^{\mathrm{c}}$ & $0.82 \pm 0.06^{\mathrm{bc}}$ & $1.06 \pm 0.19^{\mathrm{ab}}$ & $1.11 \pm 0.08^{\mathrm{a}}$ \\
低密度脂蛋白胆固醇 $/\left(\mathrm{mmol} \cdot \mathrm{L}^{-1}\right) \mathrm{LDL}$ & $3.02 \pm 0.20^{\mathrm{a}}$ & $2.28 \pm 0.29^{\mathrm{a}}$ & $2.32 \pm 0.44^{\mathrm{a}}$ & $1.84 \pm 0.45^{\mathrm{b}}$ \\
谷草转氨酶 $/\left(\mathrm{U} \cdot \mathrm{L}^{-1}\right) \mathrm{AST}$ & $81.80 \pm 3.53^{\mathrm{a}}$ & $63.40 \pm 5.34^{\mathrm{b}}$ & $48.19 \pm 5.68^{\mathrm{c}}$ & $85.97 \pm 7.62^{\mathrm{a}}$ \\
谷丙转氨酶 $/\left(\mathrm{U} \cdot \mathrm{L}^{-1}\right) \mathrm{ALT}$ & $69.23 \pm 6.26^{\mathrm{ab}}$ & $65.71 \pm 3.51^{\mathrm{b}}$ & $43.11 \pm 3.86^{\mathrm{c}}$ & $75.10 \pm 2.91^{\mathrm{a}}$ \\
碱性磷酸酶 $/\left(\mathrm{U} \cdot \mathrm{L}^{-1}\right) \mathrm{AKP}$ & $17.26 \pm 2.76^{\mathrm{a}}$ & $15.75 \pm 1.86^{\mathrm{ab}}$ & $12.08 \pm 1.15^{\mathrm{b}}$ & $13.40 \pm 2.40^{\mathrm{ab}}$ \\
\hline
\end{tabular}

注: 同行数据上标字母不同表示不同投喂频率组之间差异显著 $(P<0.05)$, 字母相同表示差异不显著 $(P>0.05)$

Note: Data in the same row with different superscript letters mean significant differences $(P<0.05)$, the same letter means that the difference is not significant $(P>0.05)$

鱼类的生长与其摄食率及饵料系数息息相 关, 摄食率和饵料系数还能反映鱼类对饲料的利 用率 ${ }^{[16]}$ 。投喂频率对鱼类生长的影响常通过 3 种方式来实现: 1) 仅提高鱼类摄食率;2) 仅提高 鱼类饲料转化率 (降低饵料系数);3) 两者同时提 高 $^{[17]}$ 。本研究中, 摄食率与饵料系数都随着投喂 频率的增加而增加, 表明长鯺篮子鱼幼鱼主要通 过摄食率的增加来促进生长, 与南方鲶 (Silurus meridionalis $)^{[18]}$ 通过提高投喂频率来增加鱼体摄 食率的结果类似。

\section{2 投喂频率对长鯺篮子鱼幼鱼生理生化的影} 响

投喂频率的改变,在影响鱼类生长的同时, 也会对鱼体的生化组成产生影响 ${ }^{[19]}$ 。研究发现, 当饲料中蛋白质含量较高时, 鱼体脂肪含量相对 稳定, 同时体内蛋白质含量随饲料摄人量的增加 而增加 ${ }^{[20]}$ 。本研究中, 各组间鱼的肌肉粗脂肪含 量无显著差异, 肌肉粗蛋白含量在 $2 \sim 4$ 次 $\cdot \mathrm{d}^{-1}$ 组间无显著差异, 表明在本研究高蛋白饲料的饲 喂下,2 次 $\cdot \mathrm{d}^{-1}$ 的投喂频率时饲料中的蛋白质 已能满足长鯺篮子鱼幼鱼所需的营养 ${ }^{[21]}$ 。

通常情况下, 当鱼体摄人的能量大于自身所 需的能量时, 会将各种能源物质转化为脂肪储存 在体内 ${ }^{[22-23]}$ 。肝脏是进行脂类代谢的重要场 所, 当血液中的游离脂肪酸含量较高时会直接将 游离脂肪酸合成脂肪 ${ }^{[24-25]}$, 因此, 血液生化指标 可以反映其营养代谢状况, 有助于研究机体的新 陈代谢及生理状况 ${ }^{[26]}$ 。本研究中, 甘油三酯含量 随着投喂频率的增加而显著增加, 且 4 次 $\cdot \mathrm{d}^{-1}$ 组血清中高密度脂蛋白含量显著增加, 低密度脂
蛋白含量显著降低, 表明在 4 次 $\cdot \mathrm{d}^{-1}$ 投喂频率 下, 长鲑篮子鱼摄人的营养物质过多, 肝脏内脂 肪开始大量累积 ${ }^{[27]}$, 王伟等 ${ }^{[28]}$ 在对花鲇 (Lateolabrax maculatus) 幼鱼的研究中也得到了 相同结果。

肝脏是血清蛋白与血脂的重要合成器官, 血 清蛋白浓度的变化可以反映机体肝脏的健康程 度 ${ }^{[29]}$ 。在本研究中, 4 次 $\cdot \mathrm{d}^{-1}$ 组的总蛋白及白 蛋白的含量相对于 3 次 $\cdot \mathrm{d}^{-1}$ 组显著下降, 表明 该投喂频率下鱼体肝脏存在一定损伤。肝脏细 胞损伤会引起细胞通透性增强, 从而释放出大量 转氨酶进人血液, 导致血液中谷丙转氨酶及谷草 转氨酶活性升高 ${ }^{[30]}$ 。在本研究中, 谷丙转氨酶及 谷草转氨酶活性在 4 次 $\cdot \mathrm{d}^{-1}$ 组中显著增加, 表 明当投喂频率达到 4 次 $\cdot \mathrm{d}^{-1}$ 时, 鱼体的肝脏细 胞因摄取过量的营养物质而导致代谢异常和细 胞损伤, 从而使血液中谷丙转氨酶及谷草转氨酶 活性升高, 这与刘伟等 ${ }^{[31]}$ 对吉富罗非鱼 (Oreochromis niloticus) 幼鱼的研究结果相似。血 清中碱性磷酸酶通常由骨骼或者肝脏释放, 通过 其活性也可以判断鱼类肝脏功能 ${ }^{[32]}$ 。血清中碱 性磷酸酶过高与机体营养不良等原因相关 ${ }^{[33]}$, 而 在本研究中, 1 次 $\cdot \mathrm{d}^{-1}$ 组的碱性磷酸酶活性显 著高于其余 3 组, 也可能与上述营养不良等原因 有关。

综合各项指标, 投喂频率 $3 \sim 4$ 次 $\cdot \mathrm{d}^{-1}$ 两组 表现出更佳的生长状况及营养指标, 再结合血液 生化指标, 可知 4 次 $\cdot \mathrm{d}^{-1}$ 组鱼的肝脏健康处于 劣势。因此,建议科学投喂频率为 3 次 $\cdot \mathrm{d}^{-1}$ 。 


\section{参考文献:}

[1] 庄 平,宋 超,章龙珍, 等. 黄斑篮子鱼肌肉营 养成分与品质的评价 $[\mathrm{J}]$. 水产学报, 2008,32 (1) :77-83.

ZHUANG P, SONG C, ZHANG $\mathrm{L} \mathrm{Z}$, et al. Evaluation of nutritive quality and nutrient components in the muscle of Siganus oramin $[\mathrm{J}]$. Journal of Fisheries of China,2008,32(1):77 - 83 .

[2]冯广朋, 章龙珍, 庄 平, 等. 海水网箱养殖长鯺 篮子鱼的摄食与生长特性 $[\mathrm{J}]$. 海洋渔业, 2008, 30 (1) : $37-42$.

FENG $\mathrm{G}$ P, ZHANG L Z, ZHUANG $\mathrm{P}$, et al. Feeding habit and growth characteristics of Siganus canaliculatus cultured in sea net cage $[\mathrm{J}]$. Marine Fisheries, 2008,30 (1) : 37 - 42 .

[3] DAUDPOTA A M, ABBAS G, KALHORO I B, et al. Effect of feeding frequency on growth performance, feed utilization and body composition of juvenile Nile tilapia, Oreochromis niloticus ( L. ) reared in low salinity water $[\mathrm{J}]$. Pakistan Journal of Zoology, 2016, 48(1): 171 - 177.

[4] TIAN H Y,ZHANG D D, LI X F, et al. Optimum feeding frequency of juvenile blunt snout bream Megalobrama amblycephala $[\mathrm{J}]$. Aquaculture, 2015 (437) :60-66.

[5] XIE D, XU S, WU Q, et al. Changes of visceral properties and digestive enzymes in the herbivorous marine teleost Siganus canaliculatus fed on different $\operatorname{diets}[\mathrm{J}]$. Acta Oceanologica Sinica , 2018, 37 (2) : $85-93$.

[6] GUO Z X, CUI J Y, LI M, et al. Effect of feeding frequency on growth performance, antioxidant status, immune response and resistance to hypoxia stress challenge on juvenile dolly varden char Salvelinus malma $[\mathrm{J}]$. Aquaculture,2018(486) : 197 - 201.

[7] DU Z Y,LIU Y J,TIAN L X,et al. The influence of feeding rate on growth, feed efficiency and body composition of juvenile grass carp ( Ctenopharyngodon idella ) [ J ]. Aquaculture International, 2006, 14(3) :247 - 257.

[8] LI M H, ROBINSON E H, OBERLE D F, et al. Effects of feeding rate and frequency on production characteristics of pond-raised hybrid catfish $[\mathrm{J}]$. North American Journal of Aquaculture, 2012, 74 (2) : $142-147$.

[9] WYSUJACK K,DRAHOTTA A. Low effect of different feeding regimes on growth and feed conversion efficiency of juvenile Eurasian perch ( Perca fluviatilis) $[\mathrm{J}]$. Aquaculture Research, 2016, 48 (9) :5166 - 5170.

[10] ERCAN K, ILHAN A, HAMZA P, et al. Effect of feeding frequency on growth, feed efficiency and nutrient utilization of juvenile flounder (Platichthys flesus luscus ) [ J ]. Aquaculture International, 2014 (2) : $723-732$.

［11］冒树泉, 邹明妤, 王春生, 等. 许氏平鲉幼鱼适宜 投喂频率的研究 $[\mathrm{J}]$. 动物营养学报, 2014,26 (8) :2379-2385.

MAO S Q, ZOU M Y, WANG C S, et al. Optimal feeding frequency for Sebastes schlegeli juveniles $[\mathrm{J}]$. Chinese Journal of Animal Nutrition, 2014,26 ( 8 ) : $2379-2385$.

[12］刘淑兰, 孙国祥, 李 杰, 等. 投喂频率对大西洋 鲑生长和生理指标的影响 $[\mathrm{J}]$. 水产科学, 2019, $38(3): 56-61$.

LIU S L, SUN G X, LI J, et al. Effects of feeding frequency on growth and some physiological indices in Atlantic salmon Salmo salar $[\mathrm{J}]$. Fisheries Science, 2019, 38(3) :56-61.

[13] 覃志彪, 赵海祥, 赵华林, 等. 投饲频率对瓦氏黄 颡鱼 (Pelteobagrus vachelh) 幼鱼胃排空、生长效益 及体组成影响 $[\mathrm{J}]$. 海洋与湖沼, 2011,42(4):506 -511 .

QIN Z B, ZHAO H X, ZHAO H L, et al. Effects of feeding frequency on gastric evacuation, growth performance and body composition of juvenile Pelteobagrus vachelh $[\mathrm{J}]$. Oceanologia et Limnologia Sinica, 2011 , 42(4) :506 - 511.

［14］刘 康,何金钊. 投喂频率和投喂水平对长吻鮠 幼鱼生长和免疫的影响 $[\mathrm{J}]$. 渔业现代化, 2019, $46(1): 3-7$.

LIU K, HE J Z. Effects of feeding frequency and feeding level on growth and immunity of juvenile Leiocassis longirostris $[\mathrm{J}]$. Fishery Modernization, $2019,46(1): 3-7$.

［15］管 敏,张德志, 饶 军, 等. 饱食投喂频率对子 二代中华鲟稚鱼生长及胃肠排空的影响 $[\mathrm{J}]$. 淡 水渔业,2019,49(4):90-97.

GUAN M, ZHANG D Z, RAO J, et al. Effects of satiation feeding frequency on growth and gastrointestinal evacuation characteristic of juvenile F2 Chinese sturgeon $[\mathrm{J}]$. Freshwater Fisheries, 2019, 49(4):90-97.

[16] 许成标. 如何降低饲料系数和提高水产养殖效益 $[\mathrm{J}]$. 饲料博览, 2016(2):52-53.

XU C B. How to reduce feed coefficient and improve 
aquaculture efficiency $[\mathrm{J}]$. Feed Review, 2016(2): $52-53$

[17] WANG N, HAYWARD R S, NOLTIE D B. Effect of feeding frequency on food consumption, growth, size variation, and feeding pattern of age-0 hybrid sunfish [J]. Aquaculture,1998,165(3):261 - 267.

[18］何利君, 谢小军, 艾庆辉. 饲喂频率对南方鲇的摄 食率、生长和饲料转化效率的影响 $[\mathrm{J}]$. 水生生物 学报,2003,27(4):434-436.

HE L J, XIE X J, AI Q H. Effects of feeding frequency on feeding rate, growth and feed conversion efficiency for the southern catfish Silurus meridioonalis $[\mathrm{J}]$. Acta Hydrobiologica Sinica, 2003, 27 (4) :434 - 436.

[19] SHEARER K D. Factors affecting the proximate composition of cultured fishes with emphasis on salmonids [J]. Aquaculture, 1994, 119 ( 1 ) : 63 88 .

[20］刘 伟,文 华,蒋 明,等. 饲料蛋白质水平与 投喂频率对吉富罗非鱼幼鱼生长及部分生理生化 指标的影响 $[\mathrm{J}]$. 水产学报, 2016,40 (5) : 751 762 .

LIU W, WEN H, JIANG M, et al. Effects of dietary protein level and feeding frequency on growth and some physiological-biochemical indexes of GIFT strain of juvenile Nile tilapia (Oreochromis niloticus) [ J ] . Journal of Fisheries of China, 2016,40(5):751 -762 .

[21] 程汉良,夏德全, 吴婷婷. 鱼类脂类代谢调控与脂 肪肝 $[\mathrm{J}]$. 动物营养学报, 2006(4):79-83.

CHENG H L, XIA D Q, WU T T. Fatty liver and regulation of lipids metabolism in fish $[\mathrm{J}]$. Chinese Journal of Animal Nutrition, 2006(4) :79 - 83 .

[22] ADIELS M, WESTERBACKA J, SORO P, et al. Acute suppression of VLDL1 secretion rate by insulin is associated with hepatic fat content and insulin resistance $[\mathrm{J}]$. Diabetologia, 2007, 50(11) : 2356 -2365 .

[23 ] ZHENG J L, LUO Z, LIU C X, et al. Differential effects of acute and chronic zinc ( $\mathrm{Zn}$ ) exposure on hepatic lipid deposition and metabolism in yellow catfish Pelteobagrus fulvidraco $[\mathrm{J}]$. Aquatic Toxicology, 2013(132 - 133):173 - 181 .

[24] XIAO L M, SHUAI L, CHAO B Q, et al. Intestinal microbiota and lipid metabolism responses in the common carp (Cyprinus carpio L. ) following copper exposure [ J ]. Ecotoxicology \& Environmental Safety, 2018(160): $257-264$.
[25] MAGKOS F. Basal very low-density lipoprotein metabolism in response to exercise: Mechanisms of hypotriacyl-glycerolemia [ J ]. Progress in Lipid Research, 2009, 48(3-4):171 - 190.

[26] 周 玉, 郭文场, 杨振国, 等. 鱼类血液学指标研 究的进展 $[\mathrm{J}]$. 上海水产大学学报, 2001,10(2): $163-165$.

ZHOU Y, GUO W C, YANG Z G, et al. Advances in the study of haemotological indices of fish $[\mathrm{J}]$. Journal of Shanghai Fisheries University, 2001, 10 (2) : $163-165$.

[27] NANTON D A, LALL S P, MCNIVEN M A. Effects of dietary lipid level on liver and muscle lipid deposition in juvenile haddock, Melanogrammus aeglefinus L. [J]. Aquaculture Research, 2001, 32 (1) : $225-234$.

[28］王 伟, 张凯强, 温海深, 等. 投喂频率对花鲇幼 鱼胃排空、生长性能和体组分的影响 $[\mathrm{J}]$. 中国海 洋大学学报(自然科学版), 2018,48(6) :55 -62.

WANG W,ZHANG K Q, WEN H S, et al. Effects of feeding frequency on gastric evacuation, growth performance and body composition of juvenile Lateolabrax maculatus [ J ]. Periodical of Ocean University of China( Natural Science Edition), 2018, $48(6)$ : $55-62$.

[29］王红梅. 血清前白蛋白在肝病中的临床意义 $[J]$. 南华大学学报 (医学版),2005,21 (1): 106 - 107.

WANG H M. Clinical significance of determining serum prealbumin in patients with viral hepatitis $[\mathbf{J}]$. Journal of University of South China (Medical Edition ) ,2005,21 ( 1 ) : 106 - 107.

[30］陈 超, 施兆鸿, 薛宝贵, 等. 低温胁迫对七带石 斑鱼幼鱼血清生化指标的影响 $[\mathrm{J}]$. 水产学报, $2012,36(8): 1249$ - 1255.

CHEN C, SHI Z H, XUE B G, et al. Influence of low-temperature stress on serum biochemical parameters in juvenile Epinephelus septemfa $[\mathrm{J}]$. Journal of Fisheries of China,2012,36 (8) : 1249 1255 .

[31］刘 伟,文 华, 蒋 明, 等. 投喂率和投喂频率 对吉富罗非鱼幼鱼生长和肝脏健康的影响 $[\mathrm{J}]$. 淡水渔业,2019,49(2):86-95.

LIU W, WEN H, JIANG M, et al. Effects of feeding rate and feeding frequency on growth performance and liver health for juvenile generically improved farmed tiplapid, Oreochromis niloticus [ J ]. Freshwater Fisheries, 2019, 49(2):86 - 95.

[32] NDREPEPA G, XHEPA E, BRAUN S, et al. 
Alkaline phosphatase and prognosis in patients with coronary artery disease $[\mathrm{J}]$. European Journal of Clinical Investigation, 2017, 47 (5) :378.

[33 ] KUNUTSOR S , BAKKER S , KOOTSTRA R, et al.
Serum alkaline phosphatase and risk of incident cardiovascular disease: Interrelationship with high sensitivity C-reactive protein $[\mathrm{J}]$. Plos One, 2015, $10(7)$ : e0132822. 


\title{
Effects of feeding frequency on growth performance, muscle composition and serum biochemical indices of juvenile Siganus canaliculatus
}

\author{
FANG Kefei ${ }^{1,2}$, LIU Jianyi ${ }^{1,2}$, WANG Yu ${ }^{1}$, FENG Guangpeng ${ }^{1,2}$, \\ ZOU Xiong ${ }^{1}$, HAO Changjie ${ }^{1,2}$, YU Yanfang ${ }^{1,2}$, SUN Xuena ${ }^{1,2}$ \\ (1. National Demonstration Center for Experimental Fisheries Science Education, Shanghai Ocean University, \\ Shanghai 201306, China; 2. Key Laboratory of Fisheries Ecology of the Yangtze Estuary, East China Sea \\ Fisheries Research Institute, Chinese Academy of Fishery Sciences, Shanghai 200090, China)
}

\begin{abstract}
The growth of fish is usually affected by the size and type of bait, feeding frequency, feeding rate and the ability of fish to absorb nutrients. Recent studies have found that feeding frequency is one of the key factors affecting the growth performance of fish. In this study, juvenile Siganus canaliculatus was used as the research object. The growth indices, muscle crude nutrients and serum biochemical indices were measured at different feeding frequencies. According to the physiological and biochemical indices of the fish body, the growth benefit under each feeding frequency was evaluated. The purpose is to find out the scientific feeding frequency for juvenile Siganus canaliculatus, and to provide the corresponding theoretical basis and scientific support for the actual production of Siganus canaliculatus and other fishes. To investigate the effects of feeding frequencies on the growth and physiology of juvenile Siganus canaliculatus, four feeding frequencies ( 1 meal $\cdot \mathrm{d}^{-1}, 2$ meals $\cdot \mathrm{d}^{-1}, 3$ meals $\cdot \mathrm{d}^{-1}, 4$ meals $\left.\cdot \mathrm{d}^{-1}\right)$ were set, marked as F1, F2, F3 and F4. Three parallel groups were set in each group for 16 weeks in a microfluidic breeding tank (effective volume $125 \mathrm{~L}$ ). Results of the study showed: 1) Growth indicators: Body weight and body length in each group increased linearly with the breeding time, the growth trend was similar between F3 and F4. The feeding rate and feed conversion ratio were the highest in $\mathrm{F} 3$, significantly higher than those of $\mathrm{F} 1 \quad(P<0.05)$. The hepatosomatic index of F4 was significantly higher than that of F1 $(P<0.05)$, and ratio of intestine weight to body weight and ratio of intestine length to body length of F1 were significantly higher than those of $\mathrm{F} 4(P<0.05)$, condition factor of F3 and F4 was significantly higher than that of F1 and F2 $(P<0.05)$. 2) Muscle crude nutrition: The muscle moisture content of F1 was significantly higher than that of the other three groups $(P<$ $0.05)$, while the muscle crude protein content of F1 was significantly lower than that of the other three groups $(P<0.05)$. The muscle crude ash content of F2 and F3 was significantly higher than that of F1 and F4 $(P<$ 0.05 ). 3 ) Serum biochemical indicators: The contents of glucose and total cholesterol were not significantly affected by feeding frequencies. The content of total protein (TP) of F2 and F3 was significantly higher than that of F1 and F4 $(P<0.05)$, while the content of albumin (ALB) of F1 and F4 was significantly higher than that of F2 and F3 $(P<0.05)$. The content of triglyceride( TG $)$ increased significantly with the breeding time $(P<0.05)$. The content of high-density lipoprotein ( HDL) was the highest in $\mathrm{F} 4$, significantly higher than that of F1 and F2 $(P<0.05)$, and the content of low-density lipoprotein ( LDL) of F4 was significantly lower than that of other three groups $(P<0.05)$. The activity of aspartate transaminase (AST) and alanine aminotransferase (ALT) was significantly lower than that of the other three groups $(P<0.05)$. Results showed that the optimal growth benefit could be gained at the feeding frequency of 3 meals $\cdot d^{-1}$.
\end{abstract}

Keywords: Siganus canaliculatus; feeding frequency; growth performance; muscle composition; serum biochemical index 